\title{
The role of lipid post-translational modification in plant developmental processes
}

\author{
Mark P. Running* \\ Department of Biology, University of Louisville, Louisville, KY, USA
}

\section{Edited by: \\ Shucai Wang, Northeast Normal \\ University, China}

Reviewed by:

Alessandro Vitale, National Research Council of Italy, Italy

Yvon Jaillais, Ecole Normale

Supérieure de Lyon, France

\section{${ }^{*}$ Correspondence:}

Mark P. Running, Department of

Biology, University of Louisville,

Louisville, KY 40292, USA

e-mail: mprunn01@louisville.edu
Most eukaryotic proteins are post-translationally modified, and modification has profound effects on protein function. One key modification is the attachment of a lipid group to certain amino acids; this typically facilitates subcellular targeting (association with a membrane) and protein-protein interactions (by virtue of the large hydrophobic moiety). Most widely recognized are lipid modifications of proteins involved in developmental signaling, but proteins with structural roles are also lipid-modified. The three known types of intracellular protein lipid modifications are S-acylation, $\mathrm{N}$-myristoylation, and prenylation. In plants, genetic analysis of the enzymes involved, along with molecular analysis of select target proteins, has recently shed light on the roles of lipid modification in key developmental processes, such as meristem function, flower development, polar cell elongation, cell differentiation, and hormone responses. In addition, while lipid posttranslational mechanisms are generally conserved among eukaryotes, plants differ in the nature and function of target proteins, the effects of lipid modification on target proteins, and the roles of lipid modification in developmental processes.

Keywords: prenylation, farnesylation, geranylgeranylation, myristoylation, acylation, palmitoylation

\section{INTRODUCTION}

With the rapid advancement of genome sequencing technologies, it is now possible to know virtually all the genes, and thus the full complement of proteins, in an increasing number of organisms. The next frontier involves learning more about these proteins: their activation/deactivation, their localization, and their function. Most proteins are post-translationally modified, and these modifications have profound implications on protein activity. This review focuses on lipid modifications of proteins. Intracellular protein lipid modifications include prenylation, $\mathrm{N}$-myristoylation, and S-acylation and serve a primary role of facilitating targeting and association of the lipidated protein to various cellular membranes and subdomains of membranes, along with a secondary role in promoting protein-protein interactions between the hydrophobic chain and a hydrophobic region of an accessory protein (Thompson and Okuyama, 2000). Hundreds of proteins are known or thought to be lipid modified in plants; this review focuses on the role of protein lipid modifications in developmental signaling and plant growth processes.

\section{PROTEIN PRENYLATION}

Of the three types of known intracellular lipid modifications of proteins in plants, protein prenylation is the most well-studied. Prenylation involves the addition of a single 15-carbon farnesyl or single or dual 20-carbon geranylgeranyl moieties to one or two cysteines near the C-terminus of target proteins (Zhang and Casey, 1996). Prenylation is conserved among Eukaryotes (Yalovsky et al., 1999; Galichet and Gruissem, 2003; Maurer-Stroh et al., 2003). Three separate heterodimeric enzymes perform prenylation: protein farnesyltransferase (PFT), protein geranylgeranyltransferase-I (PGGT), and Rab geranylgeranyltransferase (Rab-GGT, also called
geranylgeranyltransferase-II; Maurer-Stroh et al., 2003; McTaggart, 2006).

Protein farnesyltransferase and protein geranylgeranyltransferase-I share a common $\alpha$ subunit but have distinct $\beta$ subunits that are distantly related in sequence (usually $25-35 \%$ similarity) and that determine substrate specificity. PFT recognizes a C-terminal CaaX box, where $\mathrm{C}$ is the prenylated cysteine, a is usually aliphatic, and $\mathrm{X}$ is usually alanine, cysteine, glutamine, methionine, or serine. PGGT recognizes a similar sequence, except the $\mathrm{X}$ is almost always leucine. A wide range of critical signaling proteins is prenylated by PFT and PGGT, including members of the Ras superfamily of small GTPases, heterotrimeric $\mathrm{G}$ protein $\gamma$ subunits, certain classes of protein kinases, and many others (McTaggart, 2006; Maurer-Stroh et al., 2007; Nguyen et al., 2010). Mutations in the Ras family are implicated in at least $15 \%$ of human cancers, and farnesyltransferase inhibitors have undergone Phase III trials as chemotherapy agents (Nguyen et al., 2010).

Rab geranylgeranyltransferase also consists of $\alpha$ and $\beta$ subunits, which are distantly related (typically $20-30 \%$ similarity) to the PFT/PGGT $\alpha$ and $\beta$ subunits, respectively, and also requires an additional protein, Rab escort protein (REP), for activity (Leung et al., 2006). Mammalian and yeast Rab-GGT is thought to exclusively prenylate Rab GTPases, a family of Ras-related small GTPases involved in organelle biogenesis and vesicle transport (Leung et al., 2006). Rab-GGT contain a more diverse array of C-terminal target sequences, which includes dual-modified CC, CXC, CCX, CCXX, CCXXX, and the singly modified CXXX, with $\mathrm{C}$ as the modified cysteine (Maurer-Stroh and Eisenhaber, 2005). Defects in Rab-GGT prenylation are also implicated in a number of human diseases (McTaggart, 2006). 
Many studies over the years confirm that prenylation mechanisms are broadly conserved in plants (Galichet and Gruissem, 2003; Crowell and Huizinga, 2009; Sorek et al., 2009; Table 1). The $\beta$ subunits and shared $\alpha$ subunit of PFT and PGGT are present in single copy in Arabidopsis (Cutler et al., 1996; Ziegelhoffer et al., 2000; Caldelari et al., 2001; Maurer-Stroh et al., 2003; Running et al., 2004; Johnson et al., 2005). Rab-GGT is less well characterized in plants, but Rab-GGT activity has been shown to be present in extracts of tomato, tobacco, and Arabidopsis (Yalovsky etal., 1996; Hala et al., 2005). Interestingly, the Arabidopsis genome contains two putative Rab-GGT $\alpha$ and $\beta$ subunits, along with a REP homolog (Lange and Ghassemian, 2003; Maurer-Stroh et al., 2003; Hala et al., 2005, 2010; Crowell and Huizinga, 2009; Table 1); single copies of the $\alpha$ and $\beta$ subunits are present in yeast and animals examined to date (Leung et al., 2006).

\section{PFT AND PGGT FUNCTION IN PLANTS}

Genetic analysis in Arabidopsis has revealed important roles of PFT and PGGT in plant growth, development, and environmental responses. Mutations in the Arabidopsis PFT $\beta$ subunit, called enhanced response to abscisic acid1 (era1, also called wiggum), show increased sensitivity to abscisic acid (ABA) in the maintenance of seed dormancy and guard cell function (Cutler et al., 1996; Pei et al., 1998; Allen et al., 2002; Brady et al., 2003). Modulation of eral levels has been shown to preserve yield of Brassica napus (canola) under drought conditions in field trials (Wang et al., 2005). era1 mutants also have several developmental defects (Running et al., 1998; Bonetta et al., 2000; Yalovsky et al., 2000a). Shoot meristems are increased in size, resulting in altered phyllotaxy, and floral meristems are wider, resulting in extra organs, particularly extra sepals and petals. In addition, eral mutants are slightly shorter in stature and slightly slower growing. Still, the mild phenotype of eral is in contrast to homologous mutants in animals, where PFT mutants are lethal (Therrien et al., 1995; Mijimolle et al., 2005), and in yeast, where PFT is required for growth (Schafer et al., 1990).

Surprisingly, mutations in the Arabidopsis PGGT $\beta$ subunit (termed $G G B$ ) do not cause developmental defects and in fact are completely indistinguishable from wild type under normal growth conditions. Under exogenous ABA, $g g b$ mutants show slightly enhanced responses in stomata closure, and under exogenous auxin, there is a slight enhancement of lateral root formation, but no other phenotypes are apparent (Johnson et al., 2005). This is in stark contrast to animal and yeast PGGT $\beta$ subunit knockouts, which are invariably lethal (Ohya etal., 1991; Diaz etal., 1993; Trueblood etal., 1993; Therrien et al., 1995).
Mutations in the Arabidopsis a subunit shared between PFT and PGGT, termed PLURIPETALA (PLP), cause severe developmental phenotypes, including much larger shoot meristems, stem fasciation, and extra floral organs (Running et al., 2004). Flowers of $p l p$ mutants show about one extra organ per whorl, with the exception of petals, of which there can be up to 12 instead of the normal four found in wild type. The large meristems seen in $p l p$ appear to result from a defect in differentiation: primordia that are initiated from the shoot meristem occasionally fail to differentiate into a primordia fate and retain a partial or full meristem fate, initiating their own primordia and eventually fusing to the primary shoot meristem, resulting in compound shoot meristems harboring multiple central zones (Running et al., 2004). plp mutants also flower a month late under long days. plp mutants are sensitive to ABA in inhibition of seed germination, though they are not as sensitive as era1 mutants, and also show enhanced drought tolerance (Running et al., 2004; Wang et al., 2009). Adaxial leaf pavement cells have fewer lobes in plp (Sorek et al., 2011). Remarkably, plp mutants remain viable and fertile, though with reduced seed set, while PFT/PGGT $\alpha$ subunit mutants are lethal in yeast (He et al., 1991) and have not been reported in non-plant eukaryotes.

It is likely that ERA1 and GGB represent the only PFT/PGGT $\beta$ subunits in plants, as the eral ggb double mutant phenotypically resembles $p l p$ (Johnson et al., 2005). The mild phenotypes of eral and especially $g g b$ compared to $p l p$ suggest that there is a great deal of functional overlap in Arabidopsis prenylation enzymes compared to those of other eukaryotes. Cross-specificity of PFT and PGGT have been reported for a small number of target proteins in mammals and yeast, but in most cases proteins that are substrates for PFT are poor substrates for PGGT and vice versa (Zhang and Casey, 1996; Maurer-Stroh and Eisenhaber, 2005; McTaggart, 2006). Additional genetic, cytological, and biochemical evidence supports considerable cross-specificity of PFT and PGGT in plants. Overexpression of GGB in eral mutants can significantly rescue the eral phenotype (Johnson et al., 2005). Recombinant Arabidopsis PFT can prenylate several PGGT targets, including the heterotrimeric G protein $\gamma$ subunits AGG1 and AGG2, several Rop-GTPases, and selected membrane-anchored ubiquitin-fold (MUB) proteins, and farnesylation is sufficient for extensive membrane localization of most of these proteins in vivo (Downes et al., 2006; Zeng et al., 2007; Sorek et al., 2011). In vitro studies show that Arabidopsis PFT has nearly as high affinity for leucine as it does for the standard alanine, cysteine, glutamine, methionine, and serine in the $\mathrm{X}$ position of the CaaX target sequence, while PGGT shows weak but measurable affinity for non-leucine terminal amino acids (Andrews et al., 2010), which helps explain both the weak eral and very weak $g g b$ mutant phenotypes compared to $p l p$. Plant prenylation enzymes appear to be less

Table 1 | Putative protein prenylation components in Arabidopsis.

\begin{tabular}{lllll}
\hline Enzyme & $\alpha$ subunit & $\alpha$ subunitgene name(s) & $\boldsymbol{\beta}$ subunit & $\boldsymbol{\beta}$ subunitgene name(s) \\
\hline Farnesyltransferase & PFT/PGGT $\alpha$ & PLP(At3g59380) & PFT $\beta$ & $E R A 1$ (At5g40280) \\
Geranylgeranyltransferase & PFT/PGGT $\alpha$ & $P L P(A t 3 g 59380)$ & PGGT $\beta$ & GGB (At2g39550) \\
Rab geranylgeranyltransferase & Rab-GGT $\alpha$ & RGTA1, RGTA2 (At4g24490, At5g41820) & Rab-GGT $\beta$ & RGTB1, RGTB2 (At5g12210, At3g12070)
\end{tabular}


stringent in protein target sequence in general: an aromatic amino acid in the $\mathrm{a}_{2}$ position of the CaaX box does not hinder prenylation of the Rho-related GTPases Rac13 from cotton and ROP2 from Arabidopsis (Trainin et al., 1996; Johnson et al., 2005; Lane and Beese, 2006), while similar sequences act as potent inhibitors of mammalian PFT (Lane and Beese, 2006).

There are several possible explanations for the viability of plp mutants. One is the presence of non-prenylated proteins that can compensate for the loss of function of prenylated proteins. Another is that other lipid modifications, such as acylation (Hemsley and Grierson, 2008), may be enough to confer proper localization to a subset of prenylated proteins. Both of these may be true for ROP proteins, the Rho-related GTPases in plants (Vernoud et al., 2003). Arabidopsis contains 11 ROPs, 10 of which contain a CaaX motif; of these, three are either not prenylated or show only weak prenylation in vitro (Lavy etal., 2002; Zheng et al., 2002). Instead, these three (termed type II ROPs) are palmitoylated, and palmitoylation is necessary for their membrane localization and function (Lavy et al., 2002; Sorek et al., 2011).

\section{PFT AND PGGT TARGET PROTEINS}

Perhaps the most surprising aspect of the viability and fertility of plp is the sheer number of putative PFT and PGGT protein targets that would be affected in the mutant plants. Our analysis and those of others show that over 250 Arabidopsis proteins fit ideal criteria for prenylation, while as many as 700 fit minimal criteria (the presense of the cysteine four residues from the C terminus; Galichet and Gruissem, 2003). Some of the functional classes of proteins found in these database searches include transcription regulation, signaling, polar cell growth, cell cycle regulation, cell wall modification, hormone synthesis and response, metal ion homeostasis, pathogen defense response, protein folding, and many of unknown function (Galichet and Gruissem, 2003; Zeng and Running, 2008; Crowell and Huizinga, 2009; Sorek et al., 2009). Some functional classes, such as prenylated transcription factors, are not found in other eukaryotes, while some proteins that are prenylated in other systems lack prenylation motifs in plants, like nuclear lamins and inosital triphosphate $5^{\prime}$-phosphatases (Crowell and Huizinga, 2009; Sorek et al., 2009).

An increasing number of plant proteins have been shown to be prenylated in vitro, and there is direct or indirect evidence for the prenylation of several proteins in vivo as well. Of the aforementioned ROP proteins, Class I (ROP1-ROP8) contain a classical CaaL motif and are likely prenylated in vivo; indeed, ROP6 has been shown to be geranylgeranylated in vitro and in vivo (Sorek et al., 2011). Arabidopsis ROP1, ROP2, ROP3, and ROP7 were also shown to be geranylgeranylated (Sorek et al., 2009). In the absence of PGGT, ROP6 can also be farnesylated in vitro and in vivo. ROP proteins from other species have also been shown to be prenylated, including ROP1Ps from pea and Rac13 from cotton (Trainin et al., 1996).

The Arabidopsis heterotrimeric G protein $\gamma$ subunits AGG1 and AGG2 have been shown to be prenylated (Adjobo-Hermans etal., 2006; Zeng etal., 2007). Like ROP6, both AGG1 and AGG2 contain a CaaL motif for geranylgeranylation, but they can also be farnesylated in vitro. Their membrane localization is only slightly affected in a $g g b$ mutant, indicating again a compensation for the loss of PGGT by PFT, but is drastically altered in a plp mutant (Zeng et al., 2007). Interestingly, a third, atypical heterotrimeric G protein $\gamma$ subunit, AGG3, was recently identified, but, despite its plasma membrane localization, it lacks a prenylation motif and is likely not prenylated (Chakravorty et al., 2011).

There are six MUB protein family members in Arabidopsis, five of which have a CaaX or CaaL box and can be prenylated in vitro (Downes etal., 2006). All six Arabidopsis MUBs are associated with the plasma membrane in vivo. One difference observed with MUB6, which has a CaaL box, is that its plasma membrane localization is lost in a $g g b$ mutant, suggesting that PFT cannot compensate for the loss of PGGT for all target proteins.

The Arabidopsis nucleosome assembly protein AtNAP1;1 has been shown to be farnesylated in vivo (Galichet and Gruissem, 2006). AtNAP1;1 also provides the only known example for which farnesylation is developmentally regulated in plants, and nonfarnesylated AtNAP1; 1 promotes cell expansion versus cell division in later stages of leaf development (Galichet and Gruissem, 2006).

Plants also harbor the only known prenylated transcription factors, including APETALA1 protein family members (Yalovsky et al., 2000b). Arabidopsis apetalal mutant flowers show a lack of petals and a conversion of sepals to leaf-like bracts, with production of additional flowers within the flower (Mandel et al., 1992). This is different than the plp floral mutant phenotype, making it unclear what the role of prenylation is for APETALA1 function. However, prenylation of APETALA1 is required for the overexpression phenotype of a conversion of apical meristem to floral meristem fate (Yalovsky et al., 2000b).

A variety of other plant proteins have been shown to be prenylated in vitro, including the Arabidopsis metal binding domain proteins ATFP3 and HIPP26, the tobacco disease resistance-related protein NTGP4, tobacco and Arabidopsis homologs of the yeast v-SNARE protein Ykt6p, petunia calmodulin protein CaM53, the Arabidopsis AUX/IAA family member IAA4, and Arabidopsis AtIPT3 (Barth et al., 2009; Crowell and Huizinga, 2009). Disappointingly, however, there is no indication that any of the currently known prenylated proteins play any role in the striking developmental phenotype of $p l p$ mutants, suggesting that as-yet-unidentified target proteins play specific roles in meristem function and flower development.

\section{Rab-GGT FUNCTION IN PLANTS}

While PFT and PGGT mutants have been available for many years, only recently have mutants in one of the Rab-GGT subunits been characterized. Mutations in the Rab-GGT beta subunit RGTB1 gene result in a striking series of phenotypes, including smaller, epinastic leaves, loss of apical dominance (extreme branching), greatly delayed senescence, and infertility, though both male and female gametes can be effectively used in crosses (Hala et al., 2010). The mutant results in the accumulation of the unprenylated form of at least one Rab protein, Rab A2a. The mutants also show shoot gravitropic defects and a constitutive photomorphogenic 
phenotype in the dark, but surprisingly, little or no effects on root growth (Hala et al., 2010).

The effects of mutations in the other beta subunit, RGTB2, and in either of the alpha subunits RGTA1 and RGTA2, have not been reported. It would be interesting to see, for instance, if the $r g t b 1, r g t b 2$ double mutant is lethal. RGTB2 mRNA is present but is one-tenth as abundant as RGTB1 mRNA (Hala et al., 2010), suggesting that residual Rab-GGT activity may be responsible for the survivability of $r g t b 1$ mutants. Another intriguing possibility is if some Rab proteins with a CaaX motif can be efficiently prenylated by PFT or PGGT, compensating for the loss of RabGGT function. There is some precedence for this: mammalian Rab8, with a CVLL motif can be efficiently prenylated by PGGT in vitro (Wilson et al., 1998), and Rab11 (CQNI) can be prenylated by PFT (Joberty etal., 1993). Indeed, 32 of the 56 Arabidopsis Rab proteins contain a CXXX motif that can in theory act as a PFT target. Interestingly, at least one Rab protein, ARA6, is not prenylated in Arabidopsis; instead, it is myristoylated and palmitoylated (Ueda et al., 2001) and is likely to function in the absence of Rab-GGT activity. While RGTA1 is expressed everywhere, RGTA2 is expressed only in the pollen (Hala et al., 2010), suggesting that, if indeed Rab-GGT is required for survival, viable rgtal complete loss of function mutants may be more difficult to isolate.

\section{N-MYRISTOYLATION}

$\mathrm{N}$-myristoylation involves the addition of a 14 carbon saturated myristate group to the $\mathrm{N}$-terminal glycine of target proteins (Gordon et al., 1991; Johnson et al., 1994). It is carried out by a monomeric enzyme, $N$-myristoyltransferase (NMT; Duronio et al., 1989). The absolute requirement for enzymatic activity is an N-terminal glycine on the target protein, while amino acid residues two through six are used in substrate recognition, residues 7 through 10 are less restricted, and residues 11 through 17 are hydrophilic (Maurer-Stroh et al., 2002; Utsumi et al., 2004). Interestingly, experimental evidence as well as homology remodeling of NMT protein structures suggest that there are substrate specificity differences among different species (Maurer-Stroh et al., 2002).

Arabidopsis contains two homologs of NMT, termed AtNMT1 (At5g57020) and AtNMT2 At2g44170 (Qi etal., 2000), and AtNMT1 protein has been shown to have myristoylation activity on N-terminal glycine-containing peptides (Qi et al., 2000; Boisson et al., 2003; Podell and Gribskov, 2004). In fact such studies have allowed a refinement of the predicted Arabidopsis myristoylation target sequence, which is somewhat distinct from that of animals and fungi (Boisson et al., 2003; Podell and Gribskov, 2004).

Several lines of evidence indicate that AtNMT1 is the primary $\mathrm{N}$-myristoylation enzyme in Arabidopsis. Its mRNA is expressed at a higher level than that of AtNMT2 (Pierre et al., 2007), and indeed it remains unclear if AtNMT2 has any myristoylation activity, despite showing 80\% identity to AtNMT1 (Boisson et al., 2003). AtNMT1 knockouts are lethal at an early stage (Pierre et al., 2007), suggesting that AtNMT2 cannot compensate for loss of AtNMT1 activity in Arabidopsis. In addition, constitutive expression of AtNMT2 in an AtNMT1 mutant also fails to rescue (Pierre et al., 2007), indicating that AtNMT2 truly has a different function than AtNMT1. Unlike AtNMT1, AtNMT2 cannot complement a yeast $n m t 1$ mutant, and AtNMT2 cannot myristoylate at least one AtNMT1 target, SALT OVERLY SENSITIVE3 (Boisson et al., 2003).

\section{AtNMT1 AND AtNMT2 FUNCTION IN PLANTS}

A knockout of the AtNMT1 gene results in a seedling lethal phenotype: seedling arrest occurred at day 4 of germination (Pierre et al., 2007). In addition, seedlings were hypersensitive to high glucose concentration. Interestingly, the roots and root meristem were normal in this mutant, but the rib zone of the shoot meristem was completely disorganized, suggesting a role in shoot meristem establishment for AtNMT1 (Pierre et al., 2007). Lack of AtNMT1 expression at later time points shows that AtNMT1 is required for most aspects of post-embryonic development as well, including flower differentiation, fruit maturation, and fertility (Pierre et al., 2007). A weak allele of AtNMT1 shows defects in Golgi trafficking and integrity (Renna et al., 2013). The loss of AtNMT2 was much less severe than the loss of AtNMT1 and resulted strictly in a delay of flowering (Pierre et al., 2007). However, expression of AtNMT2 under the AtNMT1 promoter resulted in shoot meristem defects.

\section{MYRISTOYLATION TARGETS IN PLANTS}

As is the case for prenylation, proteins shown or predicted to be myristoylated by NMT1 Arabidopsis include several protein classes that are also myristoylated in other eukaryotes, as well as novel classes (Boisson et al., 2003; Podell and Gribskov, 2004; Martinez etal., 2008). Of the 319 Arabidopsis proteins predicted to be myristoylated by Podell and Gribskov (2004), 77 are kinases (including most of the known calcium-dependent protein kinases), 13 are phosphatases, 15 are GTP-binding proteins, 10 are transcription factors, and 132 are unknown proteins.

In recent years the number of plant proteins shown experimentally to be $\mathrm{N}$-myristoylated, either directly (by in vitro myristoylation assays) or indirectly (by mislocalization of the protein when the $\mathrm{N}$-terminal glycine is mutated) has increased significantly (Table 2). The developmental arrest of Atnmt 1 knockouts appears to be due primarily to the loss of myristoylation on SnRK1 kinase (Pierre etal., 2007), but in other cases it is unclear how these myristoylated proteins contribute to the Atnmt1 phenotype.

\section{PROTEIN S-ACYLATION}

Protein S-acylation is the covalent attachment of a fatty acid to a cysteine residue. Commonly the acyl group involved is palmitate, a 16-carbon saturated fatty acid, but other groups, including shorter or longer or unsaturated groups, can be added (Hallak et al., 1994). Unlike prenylation and N-myristoylation, S-acylation is reversible (Resh, 2006). Also unlike prenylation or myristoylation, which have specific target sequences at the $\mathrm{C}$ - and $\mathrm{N}$-terminus of the protein, the prenylated cysteine can be localized anywhere in the protein, though it is common to find acylated cysteines in close proximity to myristoylated glycines on the $\mathrm{N}$-terminus or prenylated cysteins on the C-terminus (Resh, 2006). 
Table 2 | Myristoylated proteins.

\begin{tabular}{|c|c|c|c|}
\hline Protein & Species & Function & Reference \\
\hline SnRK1 & Arabidopsis & Protein kinase & Pierre et al. (2007) \\
\hline sos3 & Arabidopsis & Calcinurin B-like protein & Ishitani etal. (2000) \\
\hline PTO & Tomato & Protein kinase & De Vries etal. (2006) \\
\hline CBL1 & Arabidopsis & Calcinurin B-like protein & Batistic et al. (2008) \\
\hline $\mathrm{NtCDPK} 2$ & Tobacco & Calcium-dependent protein kinase & Witte et al. (2010) \\
\hline CPK3 & Arabidopsis & Calcium-dependent protein kinase & Mehlmer et al. (2010) \\
\hline AtTrxh9 & Arabidopsis & h-type thioredoxin & Meng etal. (2010), Traverso et al. (2013) \\
\hline BON1 & Arabidopsis & Copine protein & Li etal. (2010) \\
\hline RPS5 & Arabidopsis & Nucleotide-binding & Takemoto et al. (2012) \\
\hline PBS1 & Arabidopsis & Protein kinase & Takemoto et al. (2012) \\
\hline POLTERGEIST & Arabidopsis & 2C-type protein phosphatase & Gagne and Clark (2010) \\
\hline POLTERGEIST-LIKE & Arabidopsis & 2C-type protein phosphatase & Gagne and Clark (2010) \\
\hline PP2C74 & Arabidopsis & 2C-type protein phosphatase & Tsugama etal. (2012b) \\
\hline PP2C52 & Arabidopsis & 2C-type protein phosphatase & Tsugama etal. (2012a) \\
\hline PCaP1 & Arabidopsis & Cation binding protein & Vijayapalani et al. (2012) \\
\hline CAN1 & Arabidopsis & Nuclease & Lesniewicz et al. (2012) \\
\hline CAN2 & Arabidopsis & Nuclease & Lesniewicz etal. (2012) \\
\hline
\end{tabular}

In contrast to prenylation or N-myristoylation, a large protein family is responsible for S-acylation. Arabidopsis harbors 24 putative protein S-acyltransferases (PATs), which are characterized by having four to six transmembrane domains and a DHHC amino acid motif within a cysteine rich domain (Hemsley and Grierson, 2008; Hemsley, 2009; Batistic, 2012). Several of these 24 have been confirmed to have acyltransferase activity (Batistic, 2012). Interestingly, the 24 PATs are localized to a variety of different intracellular membranes, although the majority is localized at the plasma membrane (Batistic, 2012).

\section{PAT FUNCTION IN PLANTS}

Despite the high number of PATs in Arabidopsis, they do not act completely redundantly; two of the PATs have been reported so far to have visible phenotypes when mutated. The first mutation in a PAT, called tip1, was originally identified in a screen for altered root hair phenotypes (Schiefelbein et al., 1993; Ryan et al., 1998). The root hairs of tip1 mutants are wider, shorter and often branched at their base. Pollen, another cell type that undergoes polar elongation, is affected in both germination and growth, resulting in reduced male fertility (Schiefelbein et al., 1993; Ryan et al., 1998). tip1 mutants also show general growth defects, including shorter stature and smaller rosettes (Ryan etal., 1998). Interestingly,
TIP1 (At5g20350) encodes the only PAT in Arabidopsis that also contains ankryin repeats, a total of six at the N-terminus; ankryin repeats play a role in protein-protein interactions (Hemsley et al., 2005).

Mutations in a second PAT, PAT10 (At3g51390), has recently been described. The PAT10 protein is localized to the tonoplast and possibly the Golgi and trans-Golgi network, and mutations in PAT10 result in pleiotropic effects on growth and environmental response (Qi et al., 2013; Zhou et al., 2013). pat10 mutants are smaller and grow much more slowly than wild type, with much smaller rosette leaves and inflorescence stems, a consequence of both reduced cell division and cell expansion (Qi et al., 2013; Zhou et al., 2013). Vascular development is also affected (Qi et al., 2013). Fertility is much reduced, a consequence of short stamens, pollen coat defects, pollen tube elongation and targeting defects, and disorganized embryo sacs (Zhou et al., 2013). pat10 mutants are also salt sensitive (Zhou et al., 2013).

\section{S-ACYLATION TARGETS IN PLANTS}

Identification of S-acylated proteins in plants using bioinformatics is not feasible due to the lack of a consensus sequence for acylation. However, a recent study using a combined proteomics/bioinformatics approach identified about 600 proteins putatively palmitoylated, much more than previously thought 
(Hemsley et al., 2013). These include a proteins with a diverse array of biochemical functions, including MAP kinases, leucinerich repeat receptor kinases, integral membrane transporters, ATPases, and SNAREs, many of which were hitherto not suspected of being acylated. This study also confirmed that S-acylation is important for the function of FLS2, a leucine-rich repeat receptor like kinase involved in pathogen perception (Hemsley et al., 2013).

Previous studies have focused on the role of S-acylation of particular target proteins. In some cases S-acylation compensates for the lack of prenylation in targeting proteins to the membranes. The three type II ROPs of Arabidopsis, AtROP9, AtROP10, and AtROP11 are S-acylated in lieu of prenylation, and S-acylation is required for their correct membrane localization (Lavy et al., 2002). AtMUB2 also is likely S-acylated via its cysteine-rich $\mathrm{C}$-terminus and is targeted to the membrane (Downes et al., 2006).

More commonly, though, S-acylation occurs in conjunction with either prenylation or myristoylation. In some examples, either prenylation or myristoylation is sufficient to target proteins to an internal membrane, while further S-acylation is required to confer plasma membrane localization. For instance, in the case of AGG2, disruption of a putative S-acylated cysteine site two amino acids upstream of the prenylated cysteine causes localization to the trans-Golgi, while full plasma membrane localization is restored when both cysteines are intact (Zeng etal., 2007). Interestingly, disruption of the putatively acylated cysteine in the same position in AGG1 does not cause a change in membrane localization (Zeng etal., 2007). Similarly, in the case of h-type thioredoxin, myristoylation alone confers localization to the ER/Golgi, and S-acylation is required for localization to the plasma membrane (Traverso et al., 2013).

Several other proteins previously mentioned are dual modified, including ARA6 (Ueda et al., 2001), type I ROPs (Sorek et al., 2007, 2011), CBL1 (Batistic et al., 2008), NtCDPK2 (Witte et al., 2010), POLTERGEIST and POLTERGEIST-LIKE (Gagne and Clark, 2010), CBL4 (Held et al., 2011), RPS5 (Qi et al., 2012), and CAN1 and CAN2 (Lesniewicz et al., 2012).

Since PAT10 and TIP1 act non-redundantly, and the other PATs cannot completely compensate for their function, it follows that at least some of their respective target proteins are unique. In the case of PAT10, it was noted that certain CBL1-related genes also have putative S-acylation sites, are also localized to the tonoplast and, like PAT10, are involved in abiotic stress responses (Zhou et al., 2013). Indeed, CBL2, CBL3, and CBL6 are all cytoplasmically localized instead of tonoplast-localized both in pat 10 mutants and when wild type plants are treated with the palmitoylation inhibitor 2-bromopalmitate, making these excellent candidates for PAT10 targets (Zhou etal., 2013). In the case of TIP1, it is possible to compare the relative S-acylation levels of a protein between wild type and tip1 mutant samples. One hundred three proteins showed a greater than or equal to 1.5 fold decrease in S-acylation in the mutant, indicating that they are either targets of TIP1 or they may show reduced acylation indirectly as a consequence of the developmental effects of tip1 mutants (Hemsley et al., 2013).

\section{CONCLUSIONS AND FUTURE PROSPECTS}

Much progress has been made in understanding the processes of protein lipidation in Arabidopsis in the past decade. In the case of prenylation and $\mathrm{N}$-myristoylation, Arabidopsis target sequences have been refined, which is important as they vary somewhat from non-plant eukaryotes, and a large scale proteomics effort have uncovered a wealth of S-acylation targets, though target sequences for S-acylation remains elusive. We now have in hand Arabidopsis mutations for many of the key lipidation enzymes, though it would be a welcome advance to differentiate a role for putative Rab-GGTase subunits RGTB2, RGTBA1, and RGTBA2, and it will be interesting to see if other PATs have phenotypes other than the two reported. It is likely that further information will come from double mutant studies; for instance, whether rgtb1 rgtb2 double mutants are lethal, and to uncover putative redundancies within the PAT family. Now that information for expression and subcellular localization of PATs are at hand (Batistic, 2012), it may be easier to identify possible redundant factors.

A wealth of information is now available for lipidated proteins; however, those that have been confirmed via in vitro and in vivo assays and subcellular localization studies still represent only a small percentage of proteins that are likely to be lipidated. More high-throughput studies on identification of prenylated or $\mathrm{N}$-myristoylated proteins would be helpful. Further characterization of additional target proteins will help clarify the multiple roles of lipidation in subcellular localization and protein function.

One gaping hole in our knowledge on plant protein lipidation is that almost all the studies of knockout and knockdown mutants in lipidation enzymes in plants has been in Arabidopsis. It is not known, for instance, if the lack of both farnesylation and geranylgeranylation (represented by plp mutants) is more generally lethal in plants (as it is in other eukaryotes), and Arabidopsis represents an outlier in its function. Similarly with the other prenylation, $\mathrm{N}$-myristoylation, or S-acylation enzymes, it is not known whether their biological function is conserved among plants. Knockouts or knockdowns of lipidation enzymes in species other than Arabidopsis will help answer these questions.

\section{ACKNOWLEDGMENT}

Work in the Running laboratory is currently funded by KSEF 2841RDE-016 to Mark P. Running.

\section{REFERENCES}

Adjobo-Hermans, M. J., Goedhart, J., and Gadella, T. W. Jr. (2006). Plant G protein heterotrimers require dual lipidation motifs of Galpha and Ggamma and do not dissociate upon activation. J. Cell Sci. 119, 5087-5097. doi: 10.1242/jcs.03284

Allen, G. J., Murata, Y., Chu, S. P., Nafisi, M., and Schroeder, J. I. (2002). Hypersensitivity of abscisic acid-induced cytosolic calcium increases in the Arabidopsis farnesyltransferase mutant era1-2. Plant Cell 14, 1649-1662. doi: 10.1105/tpc.010448

Andrews, M., Huizinga, D. H., and Crowell, D. N. (2010). The CaaX specificities of Arabidopsis protein prenyltransferases explain eral and ggb phenotypes. BMC Plant Biol. doi: 10.1186/1471-2229-10-118

Barth, O., Vogt, S., Uhlemann, R., Zschiesche, W., and Humbeck, K. (2009). Stress induced and nuclear localized HIPP26 from Arabidopsis thaliana interacts via its heavy metal associated domain with the drought stress related zinc finger transcription factor ATHB29. Plant Mol. Biol. 69, 213-226. doi: 10.1007/s11103008-9419-0 
Batistic, O. (2012). Genomics and localization of the Arabidopsis DHHC-cysteinerich domain S-acyltransferase protein family. Plant Physiol. 160, 1597-1612. doi: 10.1104/pp.112.203968

Batistic, O., Sorek, N., Schultke, S., Yalovsky, S., and Kudla, J. (2008). Dual fatty acyl modification determines the localization and plasma membrane targeting of CBL/CIPK Ca ${ }^{2+}$ signaling complexes in Arabidopsis. Plant Cell 20, 1346-1362. doi: $10.1105 /$ tpc.108.058123

Boisson, B., Giglione, C., and Meinnel, T. (2003). Unexpected protein families including cell defense components feature in the N-myristoylome of a higher eukaryote. J. Biol. Chem. 278, 43418-43429. doi: 10.1074/jbc.M307321200

Bonetta, D., Bayliss, P., Sun, S., Sage, T., and Mccourt, P. (2000). Farnesylation is involved in meristem organization in Arabidopsis. Planta 211, 182-190. doi: $10.1007 / \mathrm{s} 004250000283$

Brady, S. M., Sarkar, S. F., Bonetta, D., and Mccourt, P. (2003). The ABSCISIC ACID INSENSITIVE 3 (ABI3) gene is modulated by farnesylation and is involved in auxin signaling and lateral root development in Arabidopsis. Plant J. 34, 67-75. doi: 10.1046/j.1365-313X.2003.01707.x

Burr, C. A., Leslie, M. E., Orlowski, S. K., Chen, I., Wright, C. E., Daniels, M. J., et al. (2011). CAST AWAY, a membrane-associated receptor-like kinase, inhibits organ abscission in Arabidopsis. Plant Physiol. 156, 1837-1850. doi: 10.1104/pp.111.175224

Caldelari, D., Sternberg, H., Rodriguez-Concepcion, M., Gruissem, W., and Yalovsky, S. (2001). Efficient prenylation by a plant geranylgeranyltransferase-I requires a functional CaaL box motif and a proximal polybasic domain. Plant Physiol. 126, 1416-1429. doi: 10.1104/pp.126.4.1416

Chakravorty, D., Trusov, Y., Zhang, W., Acharya, B. R., Sheahan, M. B., Mccurdy, D. W., et al. (2011). An atypical heterotrimeric G-protein gamma-subunit is involved in guard cell $\mathrm{K}(+)$-channel regulation and morphological development in Arabidopsis thaliana. Plant J. 67, 840-851. doi: 10.1111/j.1365-313X.2011.04638.x

Crowell, D. N., and Huizinga, D. H. (2009). Protein isoprenylation: the fat of the matter. Trends Plant Sci. 14, 163-170. doi: 10.1016/j.tplants.2008.12.001

Cutler, S., Ghassemian, M., Bonetta, D., Cooney, S., and Mccourt, P. (1996). A protein farnesyl transferase involved in abscisic acid signal transduction in Arabidopsis. Science 273, 1239-1241. doi: 10.1126/science.273.5279.1239

De Vries, J. S., Andriotis, V. M., Wu, A. J., and Rathjen, J. P. (2006). Tomato Pto encodes a functional N-myristoylation motif that is required for signal transduction in Nicotiana benthamiana. Plant J. 45, 31-45. doi: 10.1111/j.1365313X.2005.02590.x

Diaz, M., Sanchez, Y., Bennett, T., Sun, C. R., Godoy, C., Tamanoi, F., et al. (1993). The Schizosaccharomyces pombe $\mathrm{cwg} 2+$ gene codes for the beta subunit of a geranylgeranyltransferase type I required for beta-glucan synthesis. EMBO J. 12, 5245-5254.

Downes, B. P., Saracco, S. A., Lee, S. S., Crowell, D. N., and Vierstra, R. D. (2006) MUBs, a family of ubiquitin-fold proteins that are plasma membrane-anchored by prenylation. J. Biol. Chem. 281, 27145-27157. doi: 10.1074/jbc.M602283200

Duronio, R. J., Towler, D. A., Heuckeroth, R. O., and Gordon, J. I. (1989). Disruption of the yeast N-myristoyl transferase gene causes recessive lethality. Science 243, 796-800. doi: 10.1126/science. 2644694

Feng, X., Shi, W., Wang, X., and Running, M. P. (2013). In vitro myristoylation assay of Arabidopsis proteins. Methods Mol. Biol. 1043, 135-139. doi: 10.1007/978-162703-532-3_14

Gagne, J. M., and Clark, S. E. (2010). The Arabidopsis stem cell factor POLTERGEIST is membrane localized and phospholipid stimulated. Plant Cell 22, 729-743. doi: 10.1105/tpc.109.068734

Galichet, A., and Gruissem, W. (2003). Protein farnesylation in plants - conserved mechanisms but different targets. Curr. Opin. Plant Biol. 6, 530-535. doi: 10.1016/j.pbi.2003.09.005

Galichet, A., and Gruissem, W. (2006). Developmentally controlled farnesylation modulates AtNAP1;1 function in cell proliferation and cell expansion during Arabidopsis leaf development. Plant Physiol. 142, 1412-1426. doi: 10.1104/pp.106.088344

Gordon, J. I., Duronio, R. J., Rudnick, D. A., Adams, S. P., and Gokel, G. W. (1991). Protein N-myristoylation. J. Biol. Chem. 266, 8647-8650.

Hala, M., Elias, M., and Zarsky, V. (2005). A specific feature of the angiosperm Rab escort protein (REP) and evolution of the REP/GDI superfamily. J. Mol. Biol.348, 1299-1313. doi: 10.1016/j.jmb.2005.02.002

Hala, M., Soukupova, H., Synek, L., and Zarsky, V. (2010). Arabidopsis RAB geranylgeranyl transferase beta-subunit mutant is constitutively photomorphogenic, and has shoot growth and gravitropic defects. Plant J. 62, 615-627. doi: 10.1111/j.1365-313X.2010.04172.x

Hallak, H., Muszbek, L., Laposata, M., Belmonte, E., Brass, L. F., and Manning, D. R. (1994). Covalent binding of arachidonate to $G$ protein alpha subunits of human platelets. J. Biol. Chem. 269, 4713-4716.

He, B., Chen, P., Chen, S. Y., Vancura, K. L., Michaelis, S., and Powers, S. (1991). RAM2, an essential gene of yeast, and RAM1 encode the two polypeptide components of the farnesyltransferase that prenylates a-factor and Ras proteins. Proc. Natl. Acad. Sci. U.S.A. 88, 11373-11377. doi: 10.1073/pnas.88.24.11373

Held, K., Pascaud, F., Eckert, C., Gajdanowicz, P., Hashimoto, K., Corratge-Faillie, C., et al. (2011). Calcium-dependent modulation and plasma membrane targeting of the AKT2 potassium channel by the CBL4/CIPK6 calcium sensor/protein kinase complex. Cell Res. 21, 1116-1130. doi: 10.1038/cr.2011.50

Hemsley, P. A. (2009). Protein S-acylation in plants (Review). Mol. Membr. Biol. 26, 114-125. doi: 10.1080/09687680802680090

Hemsley, P. A., and Grierson, C. S. (2008). Multiple roles for protein palmitoylation in plants. Trends Plant Sci. 13, 295-302. doi: 10.1016/j.tplants.2008. 04.006

Hemsley, P. A., Kemp, A. C., and Grierson, C. S. (2005). The TIP GROWTH DEFECTIVE1 S-acyl transferase regulates plant cell growth in Arabidopsis. Plant Cell 17, 2554-2563. doi: 10.1105/tpc.105.031237

Hemsley, P. A., Weimar, T., Lilley, K. S., Dupree, P., and Grierson, C. S. (2013). A proteomic approach identifies many novel palmitoylated proteins in Arabidopsis. New Phytol. 197, 805-814. doi: 10.1111/nph.12077

Ishitani, M., Liu, J., Halfter, U., Kim, C. S., Shi, W., and Zhu, J. K. (2000). SOS3 function in plant salt tolerance requires $\mathrm{N}$-myristoylation and calcium binding. Plant Cell 12, 1667-1678.

Joberty, G., Tavitian, A., and Zahraoui, A. (1993). Isoprenylation of Rab proteins possessing a C-terminal CaaX motif. FEBS Lett. 330, 323-328. doi: 10.1016/00145793(93)80897-4

Johnson, C. D., Chary, S. N., Chernoff, E. A., Zeng, Q., Running, M. P., and Crowell, D. N. (2005). Protein geranylgeranyltransferase I is involved in specific aspects of abscisic acid and auxin signaling in Arabidopsis. Plant Physiol. 139, 722-733. doi: 10.1104/pp.105.065045

Johnson, D. R., Bhatnagar, R. S., Knoll, L. J., and Gordon, J. I. (1994). Genetic and biochemical studies of protein N-myristoylation. Annu. Rev. Biochem. 63, 869-914. doi: 10.1146/annurev.bi.63.070194.004253

Lane, K. T., and Beese, L. S. (2006). Thematic review series: lipid posttranslational modifications. Structural biology of protein farnesyltransferase and geranylgeranyltransferase type I. J. Lipid Res. 47, 681-699. doi: 10.1194/jlr.R600002-JLR200 Lange, B. M., and Ghassemian, M. (2003). Genome organization in Arabidopsis thaliana: a survey for genes involved in isoprenoid and chlorophyll metabolism. Plant Mol. Biol. 51, 925-948. doi: 10.1023/A:1023005504702

Lavy, M., Bracha-Drori, K., Sternberg, H., and Yalovsky, S. (2002). A cell-specific, prenylation-independent mechanism regulates targeting of type II RACs. Plant Cell 14, 2431-2450. doi: 10.1105/tpc.005561

Lesniewicz, K., Poreba, E., Smolarkiewicz, M., Wolff, N., Stanislawski, S., and Wojtaszek, P. (2012). Plant plasma membrane-bound staphylococcal-like DNases as a novel class of eukaryotic nucleases. BMC Plant Biol. 12:195. doi: 10.1186/1471-2229-12-195

Leung, K. F., Baron, R., and Seabra, M. C. (2006). Thematic review series: lipid posttranslational modifications. geranylgeranylation of Rab GTPases. J. Lipid Res. 47, 467-475. doi: 10.1194/jlr.R500017-JLR200

Li, Y., Gou, M., Sun, Q., and Hua, J. (2010). Requirement of calcium binding, myristoylation, and protein-protein interaction for the Copine BON1 function in Arabidopsis. J. Biol. Chem. 285, 29884-29891. doi: 10.1074/jbc.M109.066100

Mandel, M. A., Gustafson-Brown, C., Savidge, B., and Yanofsky, M. F. (1992). Molecular characterization of the Arabidopsis floral homeotic gene APETALA1. Nature 360, 273-277. doi: 10.1038/360273a0

Martinez, A., Traverso, J. A., Valot, B., Ferro, M., Espagne, C., Ephritikhine, G., et al. (2008). Extent of N-terminal modifications in cytosolic proteins from eukaryotes. Proteomics 8, 2809-2831. doi: 10.1002/pmic.200701191

Maurer-Stroh, S., Eisenhaber, B., and Eisenhaber, F. (2002). N-terminal $\mathrm{N}$-myristoylation of proteins: refinement of the sequence motif and its taxonspecific differences. J. Mol. Biol. 317, 523-540. doi: 10.1006/jmbi.2002. 5425

Maurer-Stroh, S., and Eisenhaber, F. (2005). Refinement and prediction of protein prenylation motifs. Genome Biol. 6, R55. doi: 10.1186/gb-2005-6-6-r55 
Maurer-Stroh, S., Koranda, M., Benetka, W., Schneider, G., Sirota, F. L., and Eisenhaber, F. (2007). Towards complete sets of farnesylated and geranylgeranylated proteins. PLoS Comput. Biol. 3:e66. doi: 10.1371/journal.pcbi.0030066

Maurer-Stroh, S., Washietl, S., and Eisenhaber, F. (2003). Protein prenyltransferases. Genome Biol. 4, 212. doi: 10.1186/gb-2003-4-4-212

McTaggart, S. J. (2006). Isoprenylated proteins. Cell. Mol. Life Sci. 63, 255-267. doi: $10.1007 / \mathrm{s} 00018-005-5298-6$

Mehlmer, N., Wurzinger, B., Stael, S., Hofmann-Rodrigues, D., Csaszar, E., Pfister, B. et al. (2010). The $\mathrm{Ca}(2+)$-dependent protein kinase CPK3 is required for MAPKindependent salt-stress acclimation in Arabidopsis. Plant J. doi: 10.1111/j.1365313X.2010.04257.x [Epub ahead of print].

Meng, L., Wong, J. H., Feldman, L. J., Lemaux, P. G., and Buchanan, B. B. (2010). A membrane-associated thioredoxin required for plant growth moves from cell to cell, suggestive of a role in intercellular communication. Proc. Natl. Acad. Sci. U.S.A. 107, 3900-3905. doi: 10.1073/pnas.0913759107

Mijimolle, N., Velasco, J., Dubus, P., Guerra, C., Weinbaum, C. A., Casey, P. J., et al. (2005). Protein farnesyltransferase in embryogenesis, adult homeostasis, and tumor development. Cancer Cell 7, 313-324. doi: 10.1016/j.ccr.2005. 03.004

Nagasaki, N., Tomioka, R., and Maeshima, M. (2008). A hydrophilic cation-binding protein of Arabidopsis thaliana, AtPCaP1, is localized to plasma membrane via $\mathrm{N}$-myristoylation and interacts with calmodulin and the phosphatidylinositol phosphates PtdIns(3,4,5) $\mathrm{P}(3)$ and PtdIns(3,5)P(2). FEBS J. 275, 2267-2282. doi: 10.1111/j.1742-4658.2008.06379.x

Nguyen, U. T., Goody, R. S., and Alexandrov, K. (2010). Understanding and exploiting protein prenyltransferases. Chembiochem 11, 1194-1201. doi: 10.1002/cbic.200900727

Ohya, Y., Goebl, M., Goodman, L. E., Peterson-Bjorn, S., Friesen, J. D., Tamanoi, F., et al. (1991). Yeast CAL1 is a structural and functional homologue to the DPR1(RAM1) gene involved in ras processing. J. Biol. Chem. 266, 12356-12360.

Pei, Z. M., Ghassemian, M., Kwak, C. M., Mccourt, P., and Schroeder, J. I. (1998). Role of farnesyltransferase in ABA regulation of guard cell anion channels and plant water loss. Science 282, 287-290. doi: 10.1126/science.282.5387.287

Pierre, M., Traverso, J. A., Boisson, B., Domenichini, S., Bouchez, D., Giglione, C., et al. (2007). N-myristoylation regulates the SnRK1 pathway in Arabidopsis. Plant Cell 19, 2804-2821. doi: 10.1105/tpc.107.051870

Podell, S., and Gribskov, M. (2004). Predicting N-terminal myristoylation sites in plant proteins. BMC Genomics 5:37. doi: 10.1186/1471-2164-5-37

Qi, B., Doughty, J., and Hooley, R. (2013). A Golgi and tonoplast localized S-acyl transferase is involved in cell expansion, cell division, vascular patterning and fertility in Arabidopsis. New Phytol. 200, 444-456. doi: 10.1111/nph.12385

Qi, D., Deyoung, B. J., and Innes, R. W. (2012). Structure-function analysis of the coiled-coil and leucine-rich repeat domains of the RPS5 disease resistance protein. Plant Physiol. 158, 1819-1832. doi: 10.1104/pp.112.194035

Qi, Q., Rajala, R. V., Anderson, W., Jiang, C., Rozwadowski, K., Selvaraj, G., et al. (2000). Molecular cloning, genomic organization, and biochemical characterization of myristoyl-CoA:protein N-myristoyltransferase from Arabidopsis thaliana. J. Biol. Chem. 275, 9673-9683. doi: 10.1074/jbc.275.13.9673

Renna, L., Stefano, G., Majeran, W., Micalella, C., Meinnel, T., Giglione, C., etal. (2013). Golgi traffic and integrity depend on N-myristoyl transferase-1 in Arabidopsis. Plant Cell 25, 1756-1773. doi: 10.1105/tpc.113. 111393

Resh, M. D. (2006). Palmitoylation of ligands, receptors, and intracellular signaling molecules. Sci. STKE 2006, re14. doi: 10.1126/stke.3592006re14

Running, M. P., Fletcher, J. C., and Meyerowitz, E. M. (1998). The WIGGUM gene is required for proper regulation of floral meristem size in Arabidopsis. Development 125, 2545-2553.

Running, M. P., Lavy, M., Sternberg, H., Galichet, A., Gruissem, W., Hake, S., et al. (2004). Enlarged meristems and delayed growth in plp mutants result from lack of CaaX prenyltransferases. Proc. Natl. Acad. Sci. U.S.A. 101, 7815-7820. doi: 10.1073/pnas.0402385101

Ryan, E., Grierson, C. S., Cavell, A., Steer, M., and Dolan, L. (1998). TIP1 is required for both tip growth and non-tip growth in Arabidopsis. New Phytol. 138, 49-59. doi: 10.1046/j.1469-8137.1998.00896.x

Schafer, W. R., Trueblood, C. E., Yang, C. C., Mayer, M. P., Rosenberg, S., Poulter, C. D., et al. (1990). Enzymatic coupling of cholesterol intermediates to a mating pheromone precursor and to the ras protein. Science 249, 1133-1139. doi: $10.1126 /$ science. 2204115
Schiefelbein, J., Galway, M., Masucci, J., and Ford, S. (1993). Pollen tube and roothair tip growth is disrupted in a mutant of Arabidopsis thaliana. Plant Physiol. 103, 979-985. doi: 10.1104/pp.103.3.979

Sorek, N., Bloch, D., and Yalovsky, S. (2009). Protein lipid modifications in signaling and subcellular targeting. Curr. Opin. Plant Biol. 12, 714-720. doi: 10.1016/j.pbi.2009.09.003

Sorek, N., Gutman, O., Bar, E., Abu-Abied, M., Feng, X., Running, M. P., et al. (2011). Differential effects of prenylation and s-acylation on type I and II ROPS membrane interaction and function. Plant Physiol. 155, 706-720. doi: 10.1104/pp.110.166850

Sorek, N., Poraty, L., Sternberg, H., Bar, E., Lewinsohn, E., and Yalovsky, S. (2007). Activation status-coupled transient S acylation determines membrane partitioning of a plant Rho-related GTPase. Mol. Cell. Biol. 27, 2144-2154. doi: 10.1128/MCB.02347-06

Takemoto, D., Rafiqi, M., Hurley, U., Lawrence, G. J., Bernoux, M., Hardham, A. R., et al. (2012). N-terminal motifs in some plant disease resistance proteins function in membrane attachment and contribute to disease resistance. Mol. Plant Microbe Interact. 25, 379-392. doi: 10.1094/MPMI-11-10-0272

Therrien, M., Chang, H. C., Solomon, N. M., Karim, F. D., Wassarman, D. A., and Rubin, G. M. (1995). KSR, a novel protein kinase required for RAS signal transduction. Cell 83, 879-888. doi: 10.1016/0092-8674(95)90204-X

Thompson, G. A. Jr., and Okuyama, H. (2000). Lipid-linked proteins of plants. Prog. Lipid Res. 39, 19-39. doi: 10.1016/S0163-7827(99)00014-4

Trainin, T., Shmuel, M., and Delmer, D. P. (1996). In vitro prenylation of the small GTPase Rac13 of cotton. Plant Physiol. 112, 1491-1497.

Traverso, J. A., Micalella, C., Martinez, A., Brown, S. C., Satiat-Jeunemaitre, B., Meinnel, T., et al. (2013). Roles of N-terminal fatty acid acylations in membrane compartment partitioning: Arabidopsis h-type thioredoxins as a case study. Plant Cell 25, 1056-1077. doi: 10.1105/tpc.112.106849

Trueblood, C. E., Ohya, Y., and Rine, J. (1993). Genetic evidence for in vivo crossspecificity of the CaaX-box protein prenyltransferases farnesyltransferase and geranylgeranyltransferase-I in Saccharomyces cerevisiae. Mol. Cell. Biol. 13, 42604275 .

Tsugama, D., Liu, H., Liu, S., and Takano, T. (2012a). Arabidopsis heterotrimeric $\mathrm{G}$ protein beta subunit interacts with a plasma membrane $2 \mathrm{C}$-type protein phosphatase, PP2C52. Biochim. Biophys. Acta 1823, 2254-2260. doi: 10.1016/j.bbamcr.2012.10.001

Tsugama, D., Liu, S., and Takano, T. (2012b). A putative myristoylated 2C-type protein phosphatase, PP2C74, interacts with SnRK1 in Arabidopsis. FEBS Lett. 586, 693-698. doi: 10.1016/j.febslet.2012. 02.019

Ueda, T., Yamaguchi, M., Uchimiya, H., and Nakano, A. (2001). Ara6, a plantunique novel type Rab GTPase, functions in the endocytic pathway of Arabidopsis thaliana. EMBO J. 20, 4730-4741. doi: 10.1093/emboj/20.17.4730

Utsumi, T., Nakano, K., Funakoshi, T., Kayano, Y., Nakao, S., Sakurai, N., etal. (2004). Vertical-scanning mutagenesis of amino acids in a model $\mathrm{N}$-myristoylation motif reveals the major amino-terminal sequence requirements for protein N-myristoylation. Eur. J. Biochem. 271, 863-874. doi: 10.1111/j.1432-1033.2004.03991.x

Vernoud, V., Horton, A. C., Yang, Z., and Nielsen, E. (2003). Analysis of the small GTPase gene superfamily of Arabidopsis. Plant Physiol. 131, 1191-1208. doi: $10.1104 /$ pp.013052

Vijayapalani, P., Maeshima, M., Nagasaki-Takekuchi, N., and Miller, W. A. (2012). Interaction of the trans-frame potyvirus protein P3N-PIPO with host protein PCaP1 facilitates potyvirus movement. PLoS Pathog. 8:e1002639. doi: 10.1371/journal.ppat.1002639

Wang, Y., Beaith, M., Chalifoux, M., Ying, J., Uchacz, T., Sarvas, C., et al. (2009) Shoot-specific down-regulation of protein farnesyltransferase (alpha-subunit) for yield protection against drought in canola. Mol. Plant 2, 191-200. doi: $10.1093 / \mathrm{mp} / \mathrm{ssn} 088$

Wang, Y., Ying, J., Kuzma, M., Chalifoux, M., Sample, A., Mcarthur, C., et al. (2005). Molecular tailoring of farnesylation for plant drought tolerance and yield protection. Plant J. 43, 413-424. doi: 10.1111/j.1365-313X.2005.02463.x

Wilson, A. L., Erdman, R. A., Castellano, F., and Maltese, W. A. (1998). Prenylation of Rab8 GTPase by type I and type II geranylgeranyl transferases. Biochem. J. 333(Pt 3), 497-504.

Witte, C. P., Keinath, N., Dubiella, U., Demouliere, R., Seal, A., and Romeis, T. (2010). Tobacco calcium-dependent protein kinases are differentially phosphorylated in 
vivo as part of a kinase cascade that regulates stress response. J. Biol. Chem. 285, 9740-9748. doi: 10.1074/jbc.M109.052126

Yalovsky, S., Kulukian, A., Rodriguez-Concepcion, M., Young, C. A., and Gruissem, W. (2000a). Functional requirement of plant farnesyltransferase during development in Arabidopsis. Plant Cell 12, 1267-1278. doi: 10.1105/tpc.12. 8.1267

Yalovsky, S., Rodriguez-Concepcion, M., Bracha, K., Toledo-Ortiz, G., and Gruissem, W. (2000b). Prenylation of the floral transcription factor APETALA1 modulates its function. Plant Cell 12, 1257-1266. doi: 10.1105/tpc.12. 8.1257

Yalovsky, S., Loraine, A. E., and Gruissem, W. (1996). Specific prenylation of tomato Rab proteins by geranylgeranyl type-II transferase requires a conserved cysteinecysteine motif. Plant Physiol. 110, 1349-1359.

Yalovsky, S., Rodr Guez-Concepcion, M., and Gruissem, W. (1999). Lipid modifications of proteins - slipping in and out of membranes. Trends Plant Sci. 4, 439-445. doi: 10.1016/S1360-1385(99) 01492-2

Zeng, Q., and Running, M. P. (2008). "Protein lipid modification and plant development," in Floriculture, Ornamental, and Plant Biotechnology, Vol. 5, ed. J. A. Teixeira da Silva (UK: Global Science Books), 319-328.

Zeng, Q., Wang, X., and Running, M. P. (2007). Dual lipid modification of Arabidopsis Ggamma-subunits is required for efficient plasma membrane targeting. Plant Physiol. 143, 1119-1131. doi: 10.1104/pp.106. 093583

Zhang, F. L., and Casey, P. J. (1996). Protein prenylation: molecular mechanisms and functional consequences. Annu. Rev. Biochem. 65, 241-269. doi: 10.1146/annurev.bi.65.070196.001325
Zheng, Z. L., Nafisi, M., Tam, A., Li, H., Crowell, D. N., Chary, S. N., et al. (2002) Plasma membrane-associated ROP10 small GTPase is a specific negative regulator of abscisic acid responses in Arabidopsis. Plant Cell 14, 2787-2797. doi: 10.1105/tpc.005611

Zhou, L. Z., Li, S., Feng, Q. N., Zhang, Y. L., Zhao, X., Zeng, Y. L., et al. (2013). Protein S-ACYL Transferase10 is critical for development and salt tolerance in Arabidopsis. Plant Cell 25, 1093-1107. doi: 10.1105/tpc.112.108829

Ziegelhoffer, E. C., Medrano, L. J., and Meyerowitz, E. M. (2000). Cloning of the Arabidopsis WIGGUM gene identifies a role for farnesylation in meristem development. Proc. Natl. Acad. Sci. U.S.A. 97, 7633-7638. doi: 10.1073/pnas.130189397

Conflict of Interest Statement: The author declares that the research was conducted in the absence of any commercial or financial relationships that could be construed as a potential conflict of interest.

Received: 29 December 2013; paper pending published: 20 January 2014; accepted: 01 February 2014; published online: 18 February 2014.

Citation: Running MP (2014). The role of lipid post-translational modification in plant developmental processes. Front. Plant Sci. 5:50. doi: 10.3389/fpls.2014.00050

This article was submitted to Plant Cell Biology, a section of the journal Frontiers in Plant Science.

Copyright (C) 2014 Running. This is an open-access article distributed under the terms of the Creative Commons Attribution License (CC BY). The use, distribution or reproduction in other forums is permitted, provided the original author(s) or licensor are credited and that the original publication in this journal is cited, in accordance with accepted academic practice. No use, distribution or reproduction is permitted which does not comply with these terms. 